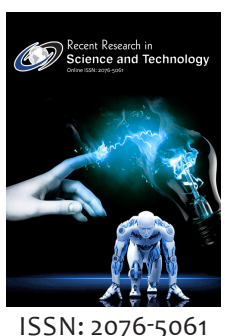

*Corresponding Author: Muhaimenul Islam Email: muhaimenulislam@iutdhaka.edu

\section{Agritouch- An efficient smartphone based approach for rooftop gardening}

\author{
Muhaimenul Islam*, Fahmid Shahriar \\ Islamic University of Technology (IUT, OIC), Department of Computer Science and Engineering, Board Bazar, \\ Gazipur 1704, Bangladesh
}

\begin{abstract}
With the increasing growth of population in mega cities like Dhaka, most of the Agricultural land has given away for housing, factories, highways and other urban needs. As a result, lack of crop production as well as various environmental crisis has already been showing up with a great threat to humankind. Rooftop gardening can be an effective method to reduce this problem and the nutritional needs of the inhabitants. But for the unavailability of the necessary resources, supports and lack of awareness among the people, most of the spaces available at the rooftop are being unutilized. We have proposed an online and smartphone-based approach to overcome the above limitations. Though the current popularity of smartphones causes rising of different mobile application technologies and selecting the appropriate one could be challenging. Here we have introduced some latest technology stack that fit best to develop our application with a comparative study of the suitable geo-location-based services. AgriTouch, a smartphone app, will act as a bridge between the gardeners and the service providers to maintain the balance of nature.
\end{abstract}

KEYWORDS: Mobile application, urban sustainability, rooftop gardening, geo-location service, cross-platform development

\section{INTRODUCTION}

Population has been increasing day by day in the urban areas of Bangladesh. Green areas have been shrinking to ensure the accommodation of mass population of the urban people. To make the capital a greener city, Dhaka City Corporation (both North and South) has recently taken a number of environment-friendly initiatives [11]. Of the many projects regarding massive plantation, one of the lucrative projects for its citizens is encouraging more people into rooftop gardening. But because of the lack of necessary resources like,

- Proper architectural plan

- Selection of plants and seeds

- Expert suggestions

- Limited information about nursery farms and product sellers

- Unavailability of seed, soil, fertilizer, insecticides etc.

People in the urban areas even having an interest in rooftop gardening can't able to involve with this movement. As a result, Effective utilization of space available at the rooftop is being minimized [2]. Urban people are deprived of the economic facilities as well as getting a pollution free environment. Also, the people who are engaged with producing and selling different agricultural products are not getting better exposure.
Different experts' knowledge is not being utilized good enough to solve our increasing environmental problems. This paper introduces a mobile app-based technology named AgriTouch, as a solution to the mentioned problems and also focuses to contribute to the selection of suitable technologies to build the application.

As, Lots of application development technologies have been published and used in various industries, choosing suitable one has direct influence on quality, cost and therefore revenue of the apps [8]. So, we have gone through some deep comparative studies on various technologies to develop our application and select the suitable ones based on expense, time and ease of development, performance, efficiency, feasibility etc. And thus, came to a decision by picking some very good technologies that are just not only meet our criteria but also introduced us to use some emerging technologies to develop our platforms. The selected technologies are also evaluated from developer point of view [5].

\section{FUNCTIONALITIES}

AgriTouch is developed to support roof top gardening related work which will be used for its outstanding service for people, 


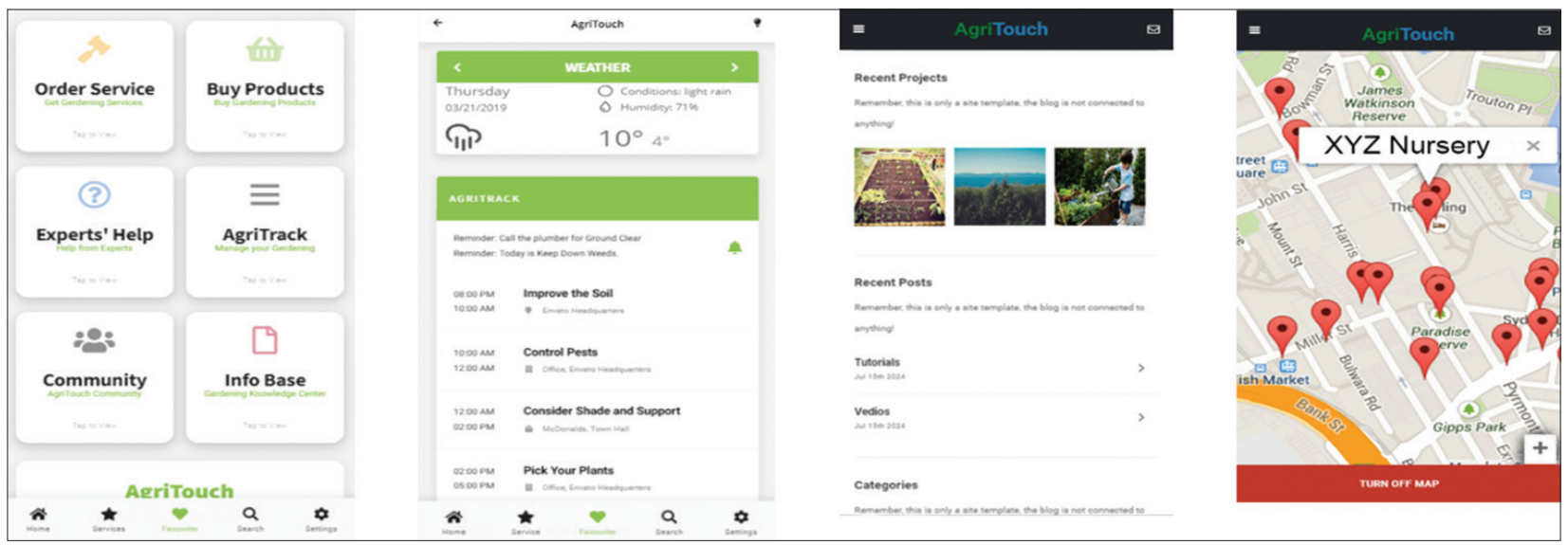

Figure 1: Functionalities of App (Main options, AgriTrack \& Weather Update, Info Base, Location Tracking)

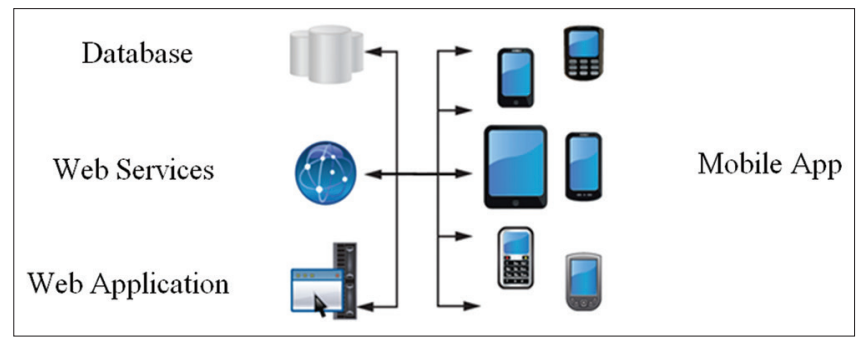

Figure 2: Complete Layer of System (REST Service)

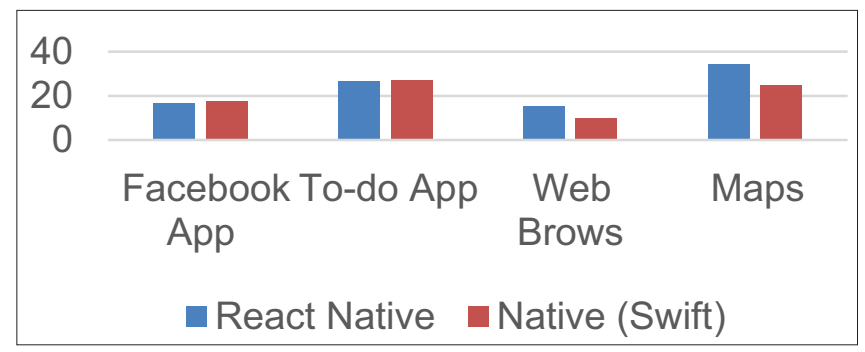

Figure 3: React Native vs Native - CPU Usage (2018)

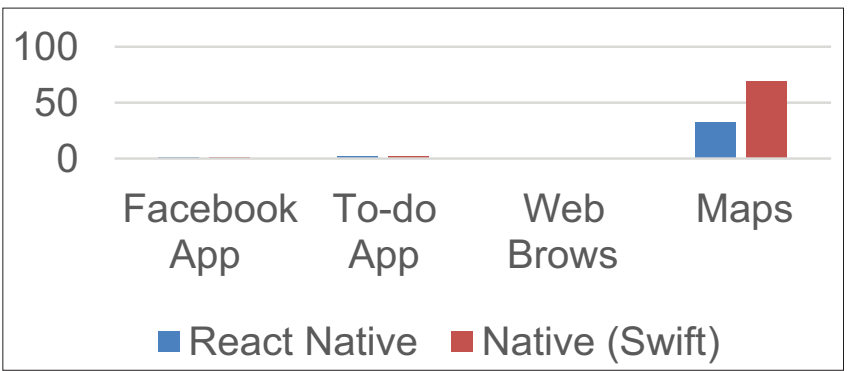

Figure 4: React Native vs Native - Memory Usage (2018)

as well as job opportunities. For users, they can get necessary information and resources regarding gardening with one tap of the phone. The nearest service provider will help the user in no time. AgriTouch is a location-based app, so it uses iOS and Android mapping and geolocation features. We should note right away that, this app is two separate applications with different functionalities,
User app - for those who are interested in rooftop gardening and need various services regarding it. This will be the main concern of this paper.

Service provider app - for those who will give architectural/ Agricultural support, get orders of the products and deliver it to the desired location.

Some main features of our apps are (Figure 1),

- Real-Time Location Tracking: Users will get location of the nurseries, agricultural products' sellers and different service provider according to their location. And also, service provider will get to know the demand of various services and their locations.

- Categories: Various categories will be available for user to search and order products and services.

- Locate and Communicate Service Provider: Users can get the location and contact information of different nurseries, gardeners, exterminators and other agricultural product sellers. User can receive the services by himself or order the service to his location.

- Communication with Experts: There will be also scope for the user to interact with the experts (Architecture/ Engineers/Agriculturists) and get their expert opinion regarding any sort of problems.

- AgriTrack and Weather Warning: User can maintain and keep track about his agricultural activities. He can also enable push notification features which will remind him about the regular basis gardening work according to his chart. Android Openweathermap API like features enables weather forecasting. Users will be notified about the weather with this app to take necessary measures to protect their garden.

- Info Base (Knowledge Center): There will be enough information, guidelines, articles and tutorials regarding roof top gardening. Information about planting, designing, utilize the roof safely and initial steps for beginner will be provided

- Payment system integration: Our app will show the services and products along with their prices. So, user can easily determine before taking the service. To reduce the payment complexity, user can pay buy few methods, like, Pay, on cash or via AgriPay, bKash, Rocket, Credit card etc.

- Rating and Feedback system: Ensure reliability by giving both users and service provider more information by 


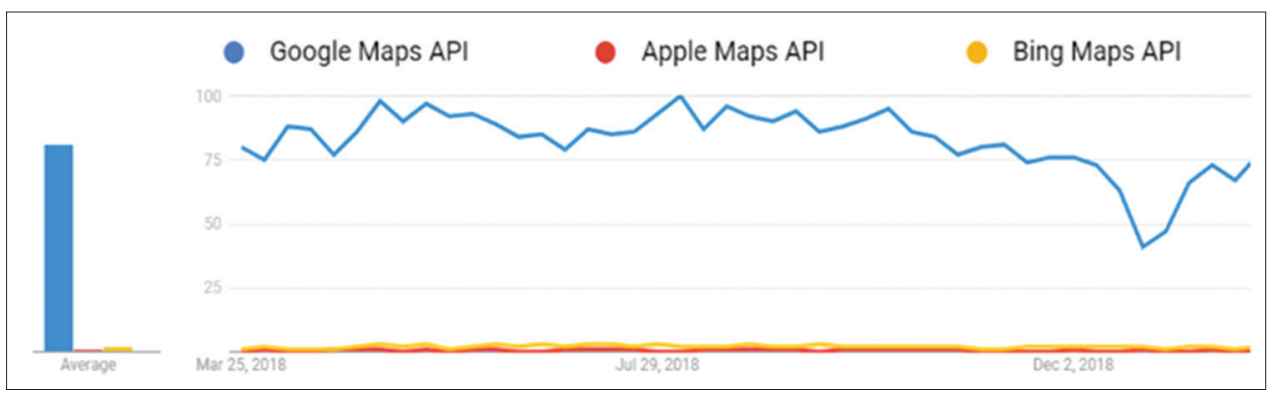

Figure 5: Interest over time comparison among Map APIs (2018)

implementing a rating system in the app. A rating system increases sense of control and security.

\section{IMPLEMENTATION}

\section{System Stacks}

Mobile App: The primary component of our system is the mobile app named as AgriTouch. It will be built in both android and iOS version because of their popularity.

Web Platform: This platform gives an opportunity for the users to use the services of AgriTouch from their web browser. Few of the features of mobile platform will be available on this platform.

As there are many user interactions, posts, service exchange, overall activities and contents need to be maintained and monitored by the admins using admin panel. Furthermore, user support, notifications, reports etc will be managed from admin web interface.

Backend Server: This will hold our overall system's backend codes and database. Backend server is responsible for communicating between our backend with web and mobile platform with API maintaining the security (Figure 2).

\section{Data Communication}

Backend of our system is implemented as a RESTful webservice. Our system will use HTTPS and Rest API to communicate and interact with each other. Thus, making it an efficient and scalable system. As most of the data communication occurs in the mobile platform, data will be fetched and refreshed in real time, we will use different 'push' services. In some special cases we will use QR code to exchange data.

\section{EFFICIENT TECHNOLOGICAL PARADIGM}

\section{Hybrid and Native}

Native app development means apps built in a particular operating system with a native language such as, Java for android apps and objective-C for IOS based apps. It takes full advantage of the operating system with great performance and user experience $[3,10]$.
Table 1: REACT native vs native vs other hybrid app

\begin{tabular}{lccc}
\hline Comparison & Native & REACT native & Other Hybrid \\
\hline Cross Platform & X & $\checkmark$ & $\checkmark$ \\
Native UI/UX & $\checkmark$ & $\checkmark$ & X \\
Performance & $\checkmark$ & $\checkmark$ & X \\
Cost \& Time & $X$ & $\checkmark$ & $\checkmark$ \\
Plugins \& Support & $\checkmark$ & $\checkmark$ & $\checkmark$ \\
\hline
\end{tabular}

On the other hand, Hybrid or Cross platform App built with various technologies (like JavaScript, or HTML5 and CSS) that's wrapped in a container allowing it to run natively on a device such as, Ionic, Xamarin, Apache Cordova etc. These apps are small web apps which are running on a browser shell in an app that has the access to the native platform layer [6].

\section{REACT Native}

It is a smooth and easy cross-platform mobile development framework for creating Android and iOS apps. With this technology, applications can be developed in JavaScript quickly and easily, and it will work as if written in native languages (Figures 3 and 4). React native uses logical components that interprets it into the necessary platform-specific element [4].

It is different from other cross-platform app development options, because React Native's abstraction layer, known as the "bridge", invokes actual Objective-C APIs or Java APIs to render to iOS components or Android components respectively instead of rendering to the browser's DOM or WebView components [7]. This component-based structure also allows to build apps with a more agile, web-style approach to development with the speed, look, and functionality of a native mobile application [9] (Table 1).

As, from these different mobile app development technologies REACT Native suits best for our situation [12].

\section{Geolocation Service}

Detect Location: Usually app uses the CoreLocation framework (iOS) or Google's Location APIs (android) to locate a user's device. This provides classes and protocols to configure and schedule location and send location events to the server. This framework also lets the app define geographic regions and monitor a device's movements, which is necessary for AgriTouch. React Native Geolocation API also provides 
these core geolocation functionalities under one hood. To use this service, react-native-geolocation-service library will be used.

Display Location: To display point-to-point routes and directions on a map within the app, usually MapKit (iOS) and Google Maps Android API (Android) is used. Through this, users will get location of the nurseries, agricultural products' sellers and different service provider according to their location. Besides Google and Apple Map API there are few maps api available to provides this feature, like Microsoft Bing Map, OpenLayers, Foursquare, OpenStreetMap, MapQuest, Here.com and so on. Each one has their own pros and cons. Because of better street view, efficient traffic condition, popularity and ease of use, we will use Google Map API for both versions of our app because, comparatively this is more matured and accurate.

Google has collected on nearly every public road since 2005. Google's Street View and Indoor Maps along with more than billion devices that use it every day makes it the most robust mapping platform (Figure 5). In our case, we will use react-native-maps React Native Map components for iOS and Android.

\section{EXPERIENCES}

Initially it would be difficult for users to get used to with the app. But it's unique and advanced experiences will attract every level of users.

User Experience: Step by step tutorial and user-friendly UI will help to learn the usage of the app. Fonts and colour combination will be modern. Menu will be self-descriptive.

Offline Service: AgriTrack and some contents of Info base will be accessible when there is no internet. Users can also access offline map.

AgriPay: This is the internal online payment system for app users. User can use this payment system regardless of any other third party payment gateways.

Advance Identification: Special image search by capturing or uploading a picture of plants or any plant diseases can be searched and identified by our advanced image recognition system.

Security and Privacy: As our app will be work with user information and monetary transaction, so sensitive information will be handled efficiently. User data will not be shared and high secured protocols will be maintained to transmit data over HTTPS.Moreover authentication, authorization and two factor authentication will ensure security.

Use of OCR: OCR gives ease of use without much interaction. We will introduce QR code functionalities in our app. Just scanning QR code, some of our services, like payment, balance recharge etc. will be available.

\section{CONCLUSION}

\section{Distribution}

After analysis and implementation phase, the distribution phase will start. Initially we will gather information about our user and service provider and store them in the server. Gradually people will register themselves, so the process is dynamic. The build version of our app will be available in the play store and also in dedicated website. We will focus on some small part of the city to initiate our project. The data collection, registration and advertisement process will be taken manually first.

\section{Impact of Solution}

Getting the available information about the resources and support of roof-top gardening by just one tap from our proposed online based system, more people will be involved in the movement. Experts' suggestion regarding roof top gardening will ensure the safety of the building and environment

One of the prominent features of our app is to let people know the economic and environmental benefit from roof-top gardening. Like the person, who will make a rooftop garden, will get a $10 \%$ holding tax rebate. Plantation of several types of vegetables and fruits, it can significantly reduce daily food costs. Furthermore, a planned and properly maintained garden on the roof can easily be a great source of earning and employment opportunities [1].

The rate at which our temperature is rising day by day, a garden on the roof of every house can help cool the air, by absorbing excessive carbon dioxide. Not just that, rooftop gardens create biodiversity in nature, through the presence of different kinds of birds and the rarest species of insects. We firmly believe our app will help to reduce the bad impact of environmental pollution and thus help to make the word a better place

\section{REFERENCES}

1. Mastura Safayet, Md. Faqrul Arefin, Md. Musleh Uddin Hasan. 2017, 'Present practice and future prospect of rooftop farming in Dhaka city: A step towards urban sustainability', Journal of Urban Management, Volume 6, pp. 56-65. [2 December 2017].

2. C. SudhakarReddy, S. Vazeed Pasha. 2016, ‘Development of national database on long-term deforestation (1930-2014) in Bangladesh', Global and Planetary Change Volume 139, pp. 173-182, National Remote Sensing Centre, Indian Space Research Organization, India.

3. Mark H. Goadrich, Michael P. Rogers. 2012, 'Smart smartphone development: iOS versus android', SIGCSE '11 Proceedings of the $42^{\text {nd }}$ ACM technical symposium on Computer science education, pp. 607-612, Dallas, TX, USA.

4. REACT Native Developer's Guide. 2019, Available from: https:// facebook.github.io/react-native

5. StackOverflow Developer Survey 2018, Available from: https:// insights.stackoverflow.com/survey/2018

6. Heitkötter H., Hanschke S., Majchrzak T.A. 2013, 'Evaluating CrossPlatform Development Approaches for Mobile Applications'. In: Cordeiro J., Krempels KH. (eds) Web Information Systems and Technologies. WEBIST 2012. Springer, Berlin, Heidelberg.

7. B Eisenman. 2015, 'Learning react native: building native mobile apps with JavaScript', O'Reilly Media, Inc.

8. Anthony I. Wasserman 2010, 'Software engineering issues for mobile application development', FoSER '10 Proceedings of the FSE/SDP 
workshop on Future of software engineering research, pp. 397-400, Carnegie Mellon Silicon Valley, USA.

9. Oleksandr Knyga, Sharmeen Hayat. 2017, React Native vs Real Native App, Available from: https://codeburst.io/react-native-vs-real-nativeapps-ad890986f1f [2 November 2017].

10. Apple Developer Connection. Accessed from: http://developer.apple. com/iphone/index.action. [6 September 2018].

11. Laila Karim 2017, Rooftop Gardening: Days of Babylon to our

NOTE: Kindly check and cite all the reference in chronological order
Current Life, Available from: https://www.thedailystar.net/lifestyle/ loving-and-living-plants/rooftop-gardening-days-babylon-our-currentlife-1378864 [21 March 2017].

12. Kishan. 2018, Why React Native is best for creating Hybrid app? (React Native vs Flutter vs Xamarin vs Ionic vs Cordova), Available from: https://www.codementor.io/kishan0/why-react-native-is-bestfor-creating-hybrid-app-react-native-vs-flutter-vs-xamarin-vs-ionic-vscordova-npqdjgcyo [36 September 2018]. 